\title{
Citations
}

\section{Silence Is Golden}

Amidst the clamor of recent RNAi papers, a quiet but intriguing trend is developing in the use of gold nanoparticles for siRNA delivery. The interest derives from evidence that gold nanoparticles can serve as a scaffold for siRNA, stabilizing it and facilitating cellular entry. Though strong levels of silencing are possible with siRNA-nanoparticle conjugates, there are applications-such as in developmental biology-for which a controlled delay between cellular entry and RNAi activity would be desirable. To that end, Braun et al. describe laser-activated release of siRNA from gold nanoparticles. Like other such conjugates, modified RNA is linked to the nanoparticles (here a hollow gold nanoshell) through a goldsulfur bond and then passivated for greater stability. However, unlike previously described siRNA-nanoparticles, the particles are subsequently coated with a lipid bilayer impregnated with Tat peptide. Decorated in this way, the complexes would be expected to enter endosomes, remaining sequestered until laser activation.
There are two steps necessary for release: the siRNA must part company with the nanoshell and then escape the endosome. Laser heating of the nanoshell is the key to both steps, initially cleaving the gold-sulfur bond and then rupturing the endosomal membrane via bubble cavitation. Although Braun et al. could not show unequivocally that siRNA release from gold nanoshells proceeded by these steps, the observed behavior was consistent with this mechanism. The degree of silencing achieved with the gold nanoshell system compared favorably with Lipofectaminemediated delivery, and cell viability remained high. Unlike more traditional methods, however, the temporal regulation achievable with the new method, combined with the possibility of spatial control via masking of laser illumination, makes this technique a golden opportunity for finely tuned silencing studies.

Braun et al. Laser-activated gene silencing via gold nanoshellsiRNA conjugates. ACS Nano [Epub ahead of print, June 15, 2009; doi: 10.1021/nn900469q].

\section{Spot Check}

Massively parallel sequencing technologies are the new standard for large-scale projects, but require careful data analysis to maximize usable information and reduce error rates. In most systems, DNA is grown, one fluorescently labeled nucleotide at a time, in arrayed clusters. As each synthesis step concludes, the system scans the surface of the imaging platform to identify a base call for each cluster. As with analysis of microarray data sets, baseline subtraction and signal correction are necessary to convert intensity values into meaningful data. Although vendors offer proprietary analysis software, an opensource alternative would be attractive to users who want maximum flexibility and direct access to the primary data and calculations for validation purposes. To meet this community need, Bioinformatics has published a paper from Whiteford et al. that introduces Swift, a tool for processing Solexa sequencing data. The program is equipped to handle fundamental image analysis issues that arise with parallel sequencing, such as proper alignment of the deck of tiled images generated as the instrument cycles. Swiff's base-calling algorithms are responsible for minimizing artifacts that can decrease yield and accuracy, such as crosstalk between the four channels, carryover from incomplete dye cleavage between cycles, and muddied signals arising from imaging a population of molecules with unavoidable variations in efficiency and accuracy of nucleotide incorporation. Compared with the commercial alternative, Swift had a marginally higher error rate, but with a nearly $14 \%$ increase in yield. The tool should be extendable to non-genomic data (such as transcriptomics) and other second-generation sequencing platforms. In addition, users wishing to benchmark their own algorithms will appreciate the authors' provision of a reference dataset. Though not yet as mature as microarray data processing methods, Swift should put the massively parallel sequencing community on the fast track to validated and optimized analysis techniques.

Whiteford et al. Swift: Primary data analysis for the Illumina Solexa Sequencing Platform. Bioinformatics [Epub ahead of print, June 23, 2009; doi: 10.1093/bioinformatics/btp383].

\section{Divide and Conquer}

One successful approach to identifying mutations rests upon cleaving DNA with a mismatch-specific endonuclease. Perhaps the best-known nuclease used for this purpose is CEL I (more familiar as SURVEYOR in the system commercialized by Transgenomic), and the readout is typically size fractionation of the cleaved and intact DNA molecules. In a paper appearing in Chemical Communications, Won et al. pair CEL I with electrochemical detection to create a new method for endonuclease-based mutation detection. The technique requires a gold electrode to which biotinylated capture probes are bound. When PCR-amplified genomic DNA anneals with the probe, mutant sequences will create a single-nucle- otide mismatch vulnerable to CEL I cleavage. Thus, the biotin moiety will be dispatched in the case of mismatched targets, but will be preserved for perfectly matched wild-type sequences. In the next step, avidin-conjugated glucose oxidase is added, followed by a biotin-modified dendrimer. At positions where the biotinylated probe remains, a glucose oxidase cluster accumulates. Glucose oxidase is a well-established tool for electrochemical detection. In real life, it oxidizes glucose to gluconolactone by means of the cofactor flavin adenine dinucleotide (FAD), which cycles back to $\mathrm{FADH}_{2}$ courtesy of oxygen; however, this function can instead be performed by the electron mediator ferrocenemethanol, which promptly donates the electrons to the electrode, resulting in measurable currents. The resulting voltammograms are detailed enough to distinguish heterozygous from homozygous wild-type and mutant samples. This new readout option should help labs using endonuclease-based genotyping embrace electrochemistry as a means to faster, easier victories in the struggle to uncover gene-disease associations.

Won et al. 2009. Mismatch DNA-specific enzymatic cleavage employed in a new method for the electrochemical detection of genetic mutations. Chem Commun (Camb). 28:4230-4232.

Selected and written by Nijsje Dorman, Ph.D. 暞

BioTechniques 47:721 (September 2009)

doi 10.2144/000113225 